\title{
LORENZO FRANCIOSINI, PRIMER TRADUCTOR DEL QUIJOTE AL ITALIANO: LOS PROBLEMAS FILOLÓGICOS DE LA PRIMERA PARTE Y EL «CASO OUDIN»
}

\section{FRANCIOSINI, LEXICÓGRAFO Y TRADUCTOR}

No existe, que yo sepa, ningún estudio específico sobre la figura de Lorenzo Franciosini en cuanto primer traductor italiano del Quijote (1622-1625). La crítica hasta ahora se ha centrado casi exclusivamente en la actividad lexicográfica de este hispanista ${ }^{1}$ y ha

\footnotetext{
${ }^{1}$ A continuación proporciono una breve bibliografía de los estudios que se han realizado hasta ahora sobre el que se puede calificar como el primer profesor conocido de lengua española que tuvo la cultura italiana. Breves noticias biograficas y criticas se encuentran en: BENEDETTO CROCE, La lingua spagnuola in Italia (Apuntes con un apéndice de Arturo Farinelli). Roma, Loescher, 1895, pp. 28-32; La Spagna nella vita italiana durante la Rinascenza, Bari, Laterza, 1968, p. 166; ALDA CROCE, «Relazioni della letteratura italiana con la letteratura spagnuola», en A.A.V.V., Letterature Comparate. Milán, Marzorati, 1948, p.122; FranCesCo Flamini, Reseña de B. CROCE, «La lingua...», en Rassegna bibliografica della letteratura italiana, IV, 1896 pp. 249-52. Noticias sobre Franciosini, sobre todo desde el punto de vista de la fonética histórica, da también Amado Alonso, De la Pronunciación Medieval a la Modema en Español. Madrid, Gredos, 1955, especialmente t. I. pp. 150-56. Sin embargo, los estudios más importantes y seminal son los de Eugenio Mele: «Per la fortuna del Cervantes in Italia nel Seicento», Studi di Filologia Modema, II, 3-4, 1909, pp. 1-27; «Tra grammatici, maestri di lingua spagnuola e raccoglitori di proverbi spagnuoli in Italiaw, Studi di Filologia Moderna, VII, 1-2, 1914, pp. 13-41; «Uno spagnolista valdesano: Lorenzo Franciosini da Castelfiorentinow, Miscellanea Storica della Valdesa, s. 64, XXII, 3, 1914, pp. 177 188. A éstos se añade el artículo de MARTIN DE RIOUER, «La obra del hispanista Lorenzo Franciosini, primer traductor del Don Quijote al italiano», Revista Nacional de Educación, II, 1942, pp. 21-28. Noticias sobre las actitud de nuestro autor
} 
reservado a su traducción tan sólo algunas aisladas o parciales alusiones - a veces felices, pero sin duda insuficientes-, a lo que entraña ${ }^{2}$. En cierto modo parece que el Franciosini traductor es un autor más consultado que leído y que su traducción ha sido objeto de lecturas tan parciales como incapaces de reconocerle dignidad autónoma al texto italiano.

Se trata, bien mirado, de un silencio doblemente culpable. Por un lado, porque no presenta correspondencia alguna en otros sectores del hispanismo europeo, en los que, en cambio, ya existen estudios específicos sobre los primeros traductores del Quijote (ej. Francia e Inglaterra) ${ }^{3}$. Por otro, porque no tiene suficientemente en

en cuanto traductor proporcionan: CESARE GREPPI, "Sulla traduzione letteraria nel Seicento italiano», Sigma, 31, 1971, pp. 59-62; PAOLO GeTreVI, Dal picaro al gentiluomo. Scrittura e immaginario nel Seicento narrativo. Milán, Franco Angeli, 1986, pp. 319-20. Se detiene en el examen de un capitulo concreto del Chisciotte de Franciosini German Colón en: Die ersten romanischen und germanischen Übersetzungen des Don Quijote (I. Teil, 16 $6^{\circ} \mathrm{Kapitel}$ ). Berna, Francke Verlag (Bibliotheca Romanica V), 1974; «Materiales para el estudio léxico contrastivo del español, del francés y del italiano: «Don Quijote» (1605) y sus traducciones coetáneas (1614 y 1622)», Travaux de Linguistique et de Litterature, 23, 1, 1985, pp. 249-293. Completan el panorama bibliográfico unos cuantos estudios especfficos sobre la actividad lexicografica de Franciosini: Annamaru Gallina, Contributi alla storia della lessicografia italo-spagnola dei secoli XVI e XVII. Florencia, Olschki, 1959, pp. 262-84; Blanca Peruoan, «La «Grammatica» de Lorenzo Franciosini», Prohemio, I, 2, 1970, pp. 225-240.

2 En su artículo de 1942 Martín de Riquer, siguiendo las sugerencias de Rodriguez Marfn (1916), afirmaba: «No hubieran divagado tanto los cervantistas sobre el significado de los «duelos y quebrantos» del principio del Don Quijote, si se hubiesen fijado, desde un principio, en el Vocabolario de Franciosini, que dice: *Comer duelos y quebrantos: $\mathrm{E}^{\prime}$ un modo di dire straordinario, e vale mangiar della carne secca con l'uova, che i Fiorenza diremmo mangiar delle frittate rognosex.» [M. DE RJoUeR, «La obra del hispanista Lorenzo Franciosini...», p. 24].

${ }^{3}$ Entre los estudios dedicados al primer traductor inglés del Quijote (1612-1620), Thomas Shelton, léase, por ejemplo, el artículo de Carmelo Cunchillos Jaime, «La primera traducción inglesa del Quijote de Thomas Shelton (1612-1620)», Cuadernos de Investigación Filológica, 9, 1-2, mayo-diciembre de 1983, pp. 63-89, con especial atención a la breve bibliografía en él citada. En cuanto a la traducción francesa de Oudin (1614) véanse por lo menos: MAURICE BARDON, «César Oudin traducteur de la $I^{\circ}$ partie du Don Quichotte (1614)», en su Don Quichotte en France aux XVII et XVIII siecles, 1605-1815. París, Champion, 1931, t. I, pp. 23-35; IsolinA SANCHEz REGUEIRA, «El hispanista francés César Oudin primer traductor de El Quijote al francés», Anales Cervantinos, XXIII, 1985, pp. 115-131. A diferencia de la obra de Franciosini, cuya ultima edición se remonta a 1832, las traducciones de Shelton y Oudin tuvieron el honor de publicarse en prestigiosas ediciones modernas que llegaron a superar el umbral del siglo xx. Constituyeron, en efecto, el patrón de la edición inglesa de James Fitzmaurice-Kelly (1896), y de las ediciones francesas de Émme Grbhur (1884) y Jean Cassou, quien en 1934 cuidó la publicación de una versión modernizada del texto de Oudin y F. de Rosset, el traductor de la segunda parte, para la célebre casa editorial Gallimard. Estos datos comprueban, una vez más, el retraso de los hispanistas italianos. 
cuenta que las dos facetas de Franciosini, la de lexicógrafo y la de traductor, constituyen las dos caras de la misma moneda en cuanto momentos distintos, pero complementarios de una global labor de mediación cultural que supone un continuado esfuerzo de traducción ${ }^{4}$.

Ahora bien, en este artículo me propongo tratar los problemas filológico-ecdócticos que la versión italiana del Quijote plantea. El examen filológico se limita tan sólo a la primera parte, de mayor interés porque nos ofrece la oportunidad para estudiar la influencia que la traducción francesa de Oudin pudo ejercer en la actividad de nuestro traductor. Para mi análisis manejo la primera edición de 1622, más útil puesto que presenta la ventaja estratégica de dejar los versos en lengua española ${ }^{5}$. El tema es, pues, muy

4 En el siglo xvi la traducción ya ha dejado de ser un mero ejercicio aristocrático, limitado exclusivamente a la aemulatio de la auctoritas clásica, y se ha convertido en un moderno fenómeno de intercambio entre distintos sistemas culturales (transistemación). La incursión del lexicógrafo Franciosini en el ámbito de la traducción literaria no es, por tanto, un episodio aislado de su actividad, sino que forma parte de esa global tarea traductora a la que nos estamos refiriendo. Toda su producción como hispanista se concentra en el breve espacio de una década: las primeras ediciones del Vocabolario, el Chisciotte, la Grammatica, los Dialogos apazibles y las Rodomuntodas son respectivamente de 1620 (Roma). La obra hispanista se concentra en el breve espacio de una década: las primeras ediciones del Vocabolario, el Chisciotte, la Grammatica, los Dialogos apazibles y las Rodomuntedas son respectivamente de 1620 (Roma), 1622 (Venecia: primera parte), 1624 (Venecia), 1625 (Venecia: segunda parte), 1626 (Venecia) y 1627 (Venecia). Los datos cronólogicos señalan una fecunda convergencia entre las obras de nuestro hispanista, que acaban así apoyándose y enriqueciéndose recíprocamente. En los años sucesivos tan sólo aparecerán nuevas ediciones. Lexicografia y experiencia traductora se intersecan: la lectura del Quijote -desde luego muy intensa, porque se añade la clara intención de regreso a la lengua propia que toda traducción suponealimenta la composición del Vocabolario a la vez que la preparación lexicográfica facilita la labor del traductor.

${ }^{5}$ En la Advertencia $A$ ' curiosi Lettori Franciosin explica su decisión con estas palabras: a... i versi non gl'ho tradotti, perche oltre all'esser difficile à chi non è Poeta; non mi son parsi tanto essenziali alla dichiarazion della prosa, che questa non si sia senz'essi, potuta volgarizzare...». El tópico, compartido a nivel europeo, al que Franciosini hace referencia es el de la imposibilidad, dificultad y/o inutilidad de la traducción del verso poético. Nuestro traductor lo interpreta de manera tan fiel que incluso renuncia a mantener en italiano la estancia del poeta napolitano Luis Tansillo que Cervantes habia traducido al espanol (cap. XXXIII).

En las ediciones posteriores se pone remedio a esta laguna. En 1625, también en Venecia y por parte del mismo editor (Andrea Baba), sale la traducción de la segunda parte del Quijote, con una dedicatoria al Ball de Volterra Ferdinando Seracinelli y con los versos traducidos por obra del poeta Alessandro Adimari. En el mismo año, probablemente como consecuencia del éxito que la primera edición habla conseguido, se volvieron a reimprimir las dos partes. Los versos se hallan traducidos al italiano y coherentemente en la advertencia a los lectores de la primera parte desaparece el pasaje relativo a las poesías del Quijote. Las dos edicio- 
específico y delimitado. Sin embargo, será capaz de suministrar sólidos elementos para empezar a bosquejar un retrato más unitario y auténtico de Franciosini.

\section{Los PROBlemas filológicos de la PRimera parte: AVeriguación DEL TEXTO DE ORIGEN}

El examen filológico de la traducción de la primera parte coincide esencialmente con la averiguación de la edición, o de las ediciones, de que pudo servirse Franciosini en su labor. Nuestro terminus ante quem es el año 1622, fecha de publicación de la primera parte del Chisciotte, exactamente doce años después de la primera edición italiana en español (Milán, 1610) y diecisiete después de las primeras ediciones en España (1605). La primera fase de nuestro trabajo, la llamada recensio, presenta, por tanto, un preciso límite cronológico, de manera que resulta posible detectar fácilmente el número de las ediciones que Franciosini pudo utilizar como texto de partida. He aquí la lista con las abreviaciones que desde ahora adoptaré para indicar cada edición a la que me refiera y los datos esenciales de cada una de ella (lugar de edición, fecha y nombre del impresor, ejemplar utilizado):

1) MADR 1. Se trata de la Editio princeps editada en Madrid por Juan de la Cuesta. Licencia, para Castilla sólo, 26 de septiembre de 1604. Utilizo la edición facsimilar realizada por la Hispanic Society of America (New York, 1905), según el ejemplar conservado en la Biblioteca Marciana de Venecia [Sign.: 86.a.127].

2) LISB 1. Lisboa. Aprobación, 26 de febrero de 1605. Por Jorge Rodriguez. [Barcelona. Biblioteca de Catalunya. Sign.: Cerv. Vit. 1-5].

3) LISB 2. Lisboa. Licencia, 27 de marzo de 1605. Por Pedro Crasbeeck. [Barcelona. Biblioteca de Catalunya. Sign.: Cerv. Vit. 1-6].

4) MADR 2. Madrid. Privilegio para Portugal, 9 de febrero de 1605. Por Juan de la Cuesta. Utilizo la reproducción facsimilar de la Hispanic Society of America (New York, 1905) según el ejemplar de la Biblioteca Marciana de Venecia [Sign.: 86.a.129].

5) VAL 1. Valencia. Aprobación, 18 de julio de 1605. Por Pedro Patricio Mey. [Barcelona. Biblioteca de Catalunya. Sign.: Cerv. Vit. 1-7].

nes (1622/1625) se publican, pues, sucesivamente y su cercanía cronológica comprueba lo prodigiosa que fue la década de los años 20-30 para la producción de Franciosini. Efectivamente, para la tercera edición habrá que esperar hasta 1677 (Roma, Giuseppe Corvo y Bartolomeo Lupandi). Ésta constituye un relativo paso atrás, porque hace referencia a la primera edición italiana $y$, por esto, deja en espanol las poesías intercaladas de la primera parte. 
6) VAL 2. Valencia. Aprobación, 18 de julio de 1605. Por Pedro Patricio Mey. [Barcelona. Biblioteca de Catalunya. Sign.: Cerv. Vit. 1-8].

7) BR 1. Bruselas. Privilegio, 7 de marzo de 1607. Por Roger Velpius. [Barcelona. Biblioteca de Catalunya. Sign.: Cerv. Vit. 1-9].

8) MADR 3. Madrid. Testimonio de las erratas, 25 de junio de 1608. Por Juan de la Cuesta. [Barcelona. Biblioteca de Catalunya. Sign.: Cerv. Vit. 1-10].

9) MIL 610. Milán. Dedicatoria, 24 de julio de 1610. Por el Heredero de Pedro Martir Locarni y Juan Bautista Bidello. [Padua. Biblioteca Civica. Sign.: L. 1620].

10) BR 2. Bruselas, 1611. Por Roger Velpius y Huberto Antonio. [Barcelona. Biblioteca de Catalunya. Sign.: Cerv. Vit. 1-13].

11) BR 3. Bruselas, 1617. Por Huberto Antonio. [Bancelona. Biblioteca de Catalunya. Sign.: Cerv. 8.VII.3].

12) BAR 617. Barcelona. Licencia, 4 de junio de 1617. Por Bautista Sorita. Constituye la primera parte de la que se puede considerar como la edición príncipe del Quijote completo: I y II Partes. [Barcelona. Biblioteca de Catalunya. Sign.: Cerv. 6.VII.11].

13) FR I. Con esta sigla indico el texto de Franciosini. Venecia, 1622. Por Andrea Baba. Dedicatoria, 13 de agosto de 1622. El ejemplar utilizado es el de la Biblioteca Nacional de Madrid [Sign.: Cerv. 1170] '

Por lo que a la selección de las variantes se refiere, cabe llevar a cabo dos observaciones preliminares, que fijan de manera llamati-

- He aquí la descripción:

L'ingegnoso Cittadino \| DON CHISCIOTTE || DELLA MANCIA.\| Composto da Michel di Cervantes Saavedra. \| Et hora nuouamente tradotto con fedeltà, e chiarezza, \| di Spagnuolo, in Italiano. \| Da Lorenzo Franciosini Fiorentino. I| Opera gustosissima, e di grandissimo trattenimento à chi è vago \| d'impiegar l'ozio in legger battaglie, disfide, incontri, amorosi || biglietti, \& inaudite prodezze di Caualieri erranti.\| Con vna Tauola ordinatissima per trouar facilmente a ogni capitolo gli $\|$ strauaganti successi e l'eroiche brauure di questo gran Caualiero. \|[Filete] \| Dedicato all'Altezza Serenissima di \| DON FERDINANDO SECONDO, \| Gran Duca di Toscana. \| [Escudo del impresor] \| IN VENETIA, Appresso Andrea Baba. MDCXXII. \| [Filete] \| Con licenza de' Superiori, \& Priuilegio.

Se trata de un vol. en $8^{\circ}$ de 11 hojas preliminares sin numerar y 669 páginas numeradas, la siguiente en blanco. Las preliminares incluyen: Portada (1. $\left.{ }^{\circ}\right)$, Dedicatoria a Ferdinando II $\left(2 .^{\circ}\right)$; Advertencia $\propto A^{\prime}$ curiosi Lettori $\left(3 .^{\circ}\right)$, el prólogo de Cervantes $\left(4 .^{\circ}-8 .^{\circ}\right)$ y la Tabla de los capítulos $\left(9 .^{\circ}-11 .^{\circ}\right)$. El ejemplar utilizado carece de las páginas 415-416 (controladas en el ejemplar de la Biblioteca Marciana. Sign. 218.C.144) y del epigrafe del capítulo XLIX (p. 627), el cual, sin embargo, se encuentra en la Tabla. Se equivoca, además, al indicar el cap. XIVII (p. 602) con la numeración del capitulo precedente, el XLVI (p. 591) -error que no se encuentra en la Tabla de los capítulos- y la página 524 por 424. 
va los límites de nuestro análisis filológico. En primer lugar, la valoración de los testimonios en cuanto y sólo como posibles fuentes inspiradoras de un trabajo de traducción forja unos criterios de investigación que no obedecen a las rígidas normas filológicas de la igualdad textual, sino que adoptan baremos mucho más elásticos. Pierden importancia las variantes ortográficas y la adquieren, en cambio, las semánticas a menos que den cabida a la corrección o interpretación autónoma del traductor. Además, la existencia de una sólida tradición filológica del texto cervantino es un dato que facilita de manera considerable el examen textual, porque ésta funciona de constante punto de referencia y permite limitar con un buen margen de seguridad el número de variantes objeto de nuestro cotejo.

De aceptar, por lo tanto, los resultados de las investigaciones llevadas a cabo por los mayores representantes de esta tradición filológica (Rius, Givanel, Suñé, Gabriel Martín del Río y Rico, Serís, Cortejón...) ${ }^{7}$, ya se puede realizar una útil clasificación por grupos, que, aunque de una forma provisional y parcial, permite reducir el número de los testimonios que tenemos que considerar.

Efectivamente, entre ellos destacan unas cuantas ediciones que no presentan variantes capaces de mermar o influir de manera determinante un trabajo de traducción y que, por consiguiente, se pueden fundamentalmente considerar copias de otras. En esencia es posible detectar dos grupos con rasgos genéticos uniformes: el grupo de Bruselas y el de Valencia.

El grupo de Bruselas incluye BR 1, BR 2 y BR 3. Las tres ediciones son básicamente copias la una de la otra. La edición de Bruselas de 1611 aes copia exacta de la de Bruselas de 1607" (Rius: I, 15) excepto la falta del soneto de Solismán y la presencia de al-

7 De ahora en adelante indicaré los catálogos bibliográficos poniendo entre paréntesis el apellido del autor (cuando haga falta), el eventual número de tomo en caracteres romanos y las páginas en números arábigos. He aquí las referencias bibliograficas completas de las obras citadas según este criterio: LEOPOLDO RIUS Y DE Lloseras, Bibliografía critica de las obras de Miguel de Cervantes Saavedra. Madrid, Libr. de M. Murillo, 1895-1904, 3 vols.; JoAN Givanel I Mas, Cataleg de la col-lecció cervantica. Barcelona, Institut d'Estudis Catalans, 1916-1925, 3 vols.; JUAN Sune Benages - Juan Sune Fonbuena, Bibliografía crítica de ediciones del Quijote impresas desde 1605 hasta 1917. Barcelona, Editorial Perello, 1917; GABRIEL-MarTIN DEL Rio y Rico, Catalogo bibliografico de la Sección de Cervantes de la Biblioteca Nacional. Madrid, Tip. de la Revista de Archivos, 1930; Homero Seris, La coleccion cervantina de la Sociedad Hispdnica de Amtrica (The Hispanic Society of America). Ediciones de Don Quijote... Urbana, University of llinois, 1918; ClemenTe Cortejón (ed.), El ingenioso hidalgo Don Quijote de la Mancha. Madrid, Victoriano Suárez, 1905-13, 6 vols.; VICENTB GaOs (ed.), El ingenioso hidalgo Don Quijote de la Mancha. Madrid, Gredos, 1987, 3 vols; Simón Dthz, Bibliografía de la Literatura hispanica. Madrid, C.S.I.C., 1970, t. VIII. 
gunas variantes ${ }^{8,}$ y la de Bruselas de 1617 «es una reimpresión exacta de la anterior del año 1611» editada, como nos explica puntualmente Rius, «con el fin de formar juego con la segunda parte publicada en 1616" (ibídem). Al grupo de Valencia pertenecen, en cambio, VAL 1, VAL 2, MIL 610 y BAR 617. A pesar de la existencia de algunas variantes en VAL 2 (Suñé, 21), las ediciones valencianas, por lo menos en esta fase inicial de la collatio, se pueden considerar como un solo testimonio: «tan leves son las diferencias tipográficas que las distinguen» (Rius: $I, 10$ ). «La diferencia más visible y conocida... está en el reclamo del recto de la segunda hoja, sin numerar, de los preliminares, que en la primera dice $L a$, y en esta [VAL 2]... Al» (Río y Rico, 15). BAR 617 hace referencia al mismo eje genealógico, puesto que se imprimió siguiendo un ejemplar valenciano de 1605 (Rius: I, 22; Suñé, 38; Giv.: I, 57), y parecidas observaciones valen también para la edición milanesa de $1610^{\circ}$. En conjunto, los testimonios que pertenecen al grupo de Valencia proceden, todos, de MADR 2, pero difieren de esta edición por la existencia de algunas variantes, que se califican, sobre todo, de adiciones ${ }^{10}$.

El segundo momento de la collatio apunta a la eliminatio de las ediciones que, a pesar de no ser meras copias unas de otras, contienen unas cuantas variantes o presentan algunos rasgos que nos impiden considerarlas como el texto de partida de la traducción de Franciosini. Es el caso del eje representado por la edición príncipe y las dos ediciones que de ella proceden, es decir, LISB 1 y LISB 2.

Muchos son los datos que impiden la procedencia del texto de Franciosini de MADR 1. Lo comprueba la presencia de los pasajes tocantes al hurto y al hallazgo del asno que sólo aparecieron desde la segunda edición de Cuesta y que Franciosini inserta adecuada-

- «Salvo algunas erratas, es copia exacta de la edición de Bruselas de 1607 » (Suné, 26). La falta del soneto no tiene mucha importancia, porque el texto de Franciosini no contiene los versos preliminares.

- Se equivoca Rrus al afimar que «esta edición, regularmente impresa, sigue estrictamente el texto de la segunda impresión de Cuestan (Rrus: I, 14; también Grv.: I, 24) y tienen en cambio razón Cortejón (CORTEJóN: I, CXIV) y su seguidor Sune (SUNE, 24-26), quien profundiza sus indicaciones, al demostrar que a la que sigue es a la de Valencia de 1605 y, sobre todo, a VAL 2 (SuNE, 25; GAOS: I, Xvii).

10 *Algunas erratas de imprenta en la segunda edición de Madrid se corrigieron en la presente [VAL 1/2], si bien otras nuevas se introdujeron. Pero deslúcenla una porción de palabras y frases anadidas caprichosamente al texto, las que no solamente son inútiles, sino que embarazan la marcha del periodo en donde se hallan ingeridas. Extrano que Salvá no hiciera mención de tales adiciones, que se notan en mayor abundancia desde el cap. XXXVIII en adelantex (Rrus: I, 9). 
mente (FR I: XXIII, 230-32; XXX, 362-63) ${ }^{11}$. Lo confirman, además, las variaciones que el traductor italiano realiza en dos pasajes de los capítulos XIX y XXVI, modificaciones que siguen el patrón de la segunda edición madrileña y que no están presentes en MADR $1{ }^{12}$.

Bastanán tan sólo estos mismos loci critici para excluir de nuestro cotejo las ediciones de Lisboa, ya que éstas en los casos examinados concuerdan básicamente con MADR 1. La historia de las dos ediciones lo demuestra de una manera aún más fehaciente. LISB 1 y LISB 2 tienen los rasgos propios de ediciones «pirata» atropelladamente impresas ante el éxito alcanzado por la edición príncipe, carente de privilegio que la amparase fuera de Castilla. Se caracterizan por mantener los defectos de incorrección tipográfica presentes en la príncipe y contener muchísimas omisiones de palabras y aún de frases enteras debidas, sobre todo, a la intervención de la Inquisición aprobante (Rius: I, 5-7). El número de las erratas y la consistencia de los expurgos presentes en estas ediciones- que Fitzmaurice-Kelly llamó atinadamente reimpresiones de pacotillafuncionaron de útiles criterios de selección de las variantes. De los casos negativos que Cortejón señala en su edición crítica (I, xxxixlx) haciendo alusión a LISB 1, me limito a recordar, como feha-

"El pasaje del cap. XXIII empieza con «Aquella noche llegaron a la mitad de las entranas de Sierra Morena.... y finaliza «Consolose Sancho con esto; y limpio sus lagrimas, têmplo sus solloços, y agradecio a Don Quixote la merced que le hazia. El qual como entró por aquellas montanas...» (fol. 108). Franciosini traduce: «Quella sera giunsero nel mezzo di Sierra Morena... Con questa promessa, Sancio si consold, rasciugd le lacrime, messe freno a singulti, \& ringrazio per tanto fauore don Chrisciotte... (XXIII, 230-32). El segundo pasaje empieza con «Mientras esto passaua y finaliza con «Sancho se lo agradecio» (fol, 171), a lo cual corresponde en el texto italiano $\ll$ Stando in questi ragionamenti....Sancio le rese infinite grazie...» (XXX, 362-63).

12 El pasaje del cap. XIX inícia en la edición príncipe con «Olvidauaseme de dezir, que aduierta vuestra merced, q. queda descomulgado...* (fol. 84). MADR 2 lo modifica en \&... y dixole: Yo entiendo Sancho, que quedo descomulgado...» (fol. 86) y FR I acepta el cambio: «... lo credo Sancio, d'esser rimasto scomunicato...» (180). El pasaje del cap. XXVI empieza con «se hartó de llorar y de encomendarse a Dios... y acaba con «vn milló de Aue Marias» (fol. 132). MADR 2 lo sintetiza y atenúa los pormenores referentes al rosario (fol. 132). FR I se conforma puntualmente con esta versión más reducida (280-281). Por evidentes razones de síntesis no puedo citar los pasajes de forma integral. La limitación no vale solamente en este caso concreto. Por lo general, a lo largo de este artículo me limitare a recordar tan sólo algunas de las variantes significativas que tuve ocasión de detectar durante mi labor de investigación. Para otros casos de igual relieve y otras variantes, a lo mejor no intrínsecamente fehacientes, pero igualmente capaces, tomadas en su conjunto, de avalar mis afirmaciones remito a mi tesis de licenciatura intedita: La obra de LORENZO FRANCIOSINI: Aproximación a la primera traducción italiana del «Quijote» (1622-25). Estudio de la primera parte. Universidad de Venecia, Año Ạcadémico 1990-91. 
ciente la supresión, en la Canción de Grisóstomo, del terceto que empieza por «El rugir del león» y termina por «el espantable» y de los versos comprendidos entre "Del ya vencido toro el implacable" y "Con lenguas muertas y palabras vivas»: lagunas, éstas, que no tienen correspondencia en el texto de Franciosini. En cuanto a LISB 2 valen las mismas observaciones, ya que «se notan los mismos defectos de incorrección tipográfica y omisiones que en la anterior de Rodríguez, y son aún más frecuentes las últimas» (Rius: I, 7).

Por otra parte, además de los datos procedentes del examen textual, la exclusión del eje genético que hace referencia a MADR 1 queda valorizada por la escasa fortuna que tuvo la primera edición de Cuesta ${ }^{13}$ y por la existencia de una fuerte censura que impidió que las ediciones de Lisboa se difundieran en el extranjero. Se desprende, por tanto, que la edición de partida que Franciosini hubo de utilizar se inserta necesariamente en la línea genealógica que tiene por texto matriz la segunda edición madrileña de Cuesta.

De los testimonios que de ésta proceden MADR 3 es el que potencialmente reuniría los requisitos necesarios para aspirar al honor de texto-fuente, porque en él destacan algunas significativas convergencias que no todas las demás ediciones presentan ${ }^{14}$. Sin embargo, mucho más llamativos son los casos que divergen del texto de Franciosini, casos que, por su difusión, nos inducen a excluir esta edición del árbol genealógico. Me limito a citar algunos loci critici para señalar las tres directrices de la divergencia textual (1. Omisión; 2. Adición; 3. Lectio distinta):

1 CAP. XLVIII. Fol. 292.

MADR 2 ... que de milagros falsos fingen en ellas...

MADR 3 ... que de milagros fingen en ellas...(255r).

FR I ... o quanti miracoli falsi, che vi fingono... (621).

2. CAP XXXV. Fol 212v.

13 «Exceptuando las dos ediciones de Lisboa, todas las que se han impreso durante más de 250 años siguen el texto de la segunda; lo que demuestra el hecho, difícil de explicar, de haber en seguida desaparecido los pocos ejemplares que de la edición príncipe se tiranon.» (Rius: 1,2 ). Resume las posibles explicaciones Simón Dinz: $\alpha$ La desaparición rápida y casi total de los ejemplares se ha atribuido por unos al deseo de enmendar los errores y por Rodriguez Marín al envío a América de la mayor parte de la tirada, aunque otros afirman que por la fecha de los embarques muchos de estos ejemplares podían ser ya de la segunda edición» (SIMON DiAz: VIII 27).

${ }^{14}$ Tan sólo un ejemplo. CAP. XXV. Fol. 130 MADR 2... por otros tantos aqui recebidos de contado, que consta, y con su carta de pago seran bien dados...; MADR 3... que cô esta seran bien dados... (114v); FR I ... per altrettanti riceuuti qui di contanti, che con questa, e con la sua riceuuta saranno ben dati... (276). 
MADR 2 ... q. claramente conocio que se le yua acabando la vida...

MADR 3....que claramente conocio por las premisas mortales que en si sentia que se le iua acabando la vida...(186v).

FR I ... che conobbe chiaramente, che a poco a poco s'andaua consumando...(451).

3. CAP. XXV. Fol. 130.

MADR 2 ... Fecha en las entranas de Sierra Morena, a veynte y dos de Agosto, deste presente año...

MADR 3 ... a veinte, y siete Agosto...(114v).

FR I ... Data nelle viscere di Sierra Morena a ventidua d'Agosto di questo present'anno... (276).

En las muestras que acabamos de considerar Franciosini se aleja de MADR 3 y acepta la lectio de MADR 2. Esta edición podría, por lo tanto, aspirar a ser nuestro texto-fuente $y$, efectivamente, no cabe duda de que un control directo de la traducción italiana comprobaria la presencia de muchas variantes positivas. No obstante, el dato no es de por sí fehaciente: de esta edición dependen, en efecto, todas las demás y, consiguientemente, no sería de extrañar que muchas de esas mismas variantes positivas se encontraran en las ediciones ahijadas. La exclusión de MADR 2 de la lista de los posibles «candidatos» es, en cierto modo, un resultado que se impone indirecta, pero necesariamente a la hora de tomar en consideración los testimonios que nos quedan por examinar.

Rius no duda de alguna forma en afirmar que «Franciosini tomó por original la edición de Bruselas, del año 1607; por esto se encuentran en esta traducción las variantes que dicha edición introdujo referentes á la pérdida del rucio en Sierra Morena» (I, 301). El cotejo que llevamos a cabo comprueba esta afirmación o, mejor dicho, que Franciosini tuvo que conocer una de las ediciones de Bruselas ${ }^{15}$. He aquí algunas de las variantes relacionadas con el robo del rucio:

CAP. XXIII. Fol. 109.

MADR 2 ... y assi yua tras su amo, sentado a la mugeriega sobre su jumento, sacando de vn costal y embaulando en su paça...

BR 1 ... y asi iua tras su amo, sacando de quando en quando de vn costal (que Rozinante lleuaua sobre si por falta del asno), y embaulando en su pança... (210)

15 Si se excluye una diferencia de paginación que distingue BR 1 de BR $2 / 3$, en los casos que desde ahora se van a examinar los tres testimonios coinciden, de forma que, por lo menos de momento, sigo a Rius y tomo como edición de referencia la de Bruselas de 1607. 
FR I ... e cosi se n'andaua dietro al suo padrone, cauando di quando in quando d'un sacco, (che Ronzinante portaua per non ci esser l'Asino), \& empiendo la pancia... (232).

CAP. XXIII. Fol. 112.

MADR 2 ... y siguiole Sancho con su acostumbrado jumêto...

BR 1 ... y siguiole Sancho a pie consolado de la perdida de su jumento con la esperança de los tres pollinos... (216).

FR I ... e Sancho lo seguito a piedi; consolato della perdita del suo giumento per la speranza de tre polledri... (238)

CAP. XXV. Fol. 121.

MADR 2 ... porque departiera yo con mi jumento lo que me viniera en gana...

BR 1 ... porque departiera yo con Rozinante (ya que mi corta ventura no permitio pueda ser con mi jumento) lo que me viniera en gana... (233).

FR I ... che io potrei dire con Ronzinâte, (già che la mia cattiua disgrazia non ha voluto ch'io possa far questo col mio giumento) quello che mi veniBe alla fantasia... (257).

Otros datos refuerzan la procedencia de FR I del gnupo de Bruselas. De este grupo Franciosini acepta, por ejemplo, la feliz transposición de los epígrafes de los cap. XXXV y XXXVI, que en las ediciones de Cuesta y sus ahijadas estaban, en cambio, trocados (Rius: I, 12) ${ }^{16}$. A esta acertada corrección se suman otras variantes, importantes, en primer lugar, porque, al distribuirse a lo largo de todo el texto de Franciosini, descartan la hipótesis de una presencia ocasional del grupo de Bruselas, y, en segundo, por la convergencia entre MADR 2, MADR 3 y el grupo valenciano, significativa exactamente porque confirma de forma inequívoca la dependencia de FR I de BR 1. Unos cuantos ejemplos:

CAP. XXI. Fol. 98v.

MADR 2 ... Consuelase con esto la cuytada, y procura consolarse...

16 BR 1. CAP. XXXV. Donde se da fin a la nouela del curioso impertinente, $y$ se cuenta la braua y descomunal batalla, que Don Quijote tuuo con unos cueros de vino tinto. (396) FR I. CAP. XXXV. Nel quale si da fine alla nouella del Curioso Impertinente, e si racconta vna braua, e sanguinosa battaglia, che Don Chisciotte fece con certi otri di vino rosso. (441) BR 1. CAP. XXXVI. Que trata de otros raros sucesos, que en la venta sucedieron. (406) FR I. CAP. XXXVI. Che tratta d'altri rari casi, che successero nell'hosteria. (453) La edición de Milán, por ejemplo, sigue la sucesión de MADR 2. MIL 610. CAP. XXXV. Donde se da fin a la nouela del curioso impertinente. (476); MIL 610. CAP. XXXVI. Que trata de la braua, y descomunal batalla que Don Quixote tuuo con unos cueros de vino tinto, con otros raros sucesos que en la venta sucedieron. (489). 
BR 1 ... consuelase con esto la cuitada, y procura alegrarse... (1.90).

FR I ... L'infelice si consola con questo, e procura di rallegrarsi... (208). EPITAFIO O SONETO DEL MONICONGO [Fol. 314v.]

MADR 2. El caluatrueno, que adornó a la Mancha, / De mas despojos que Jason decreta...

BR 1. El caluatrueno que adorno á la Mancha / De más despojos que Jason de Creta... (592).

FR I. El Caluatrueno que adorno la Mancha / De mas despojos que Iason de Creta... (666).

Sin embargo, ¿de verdad Franciosini sigue el patrón de BR 1, como afirma Rius? De las tres ediciones de Bruselas, ya se ha dicho que BR 2 es una copia, salvo algunas variantes, de BR 1 y que BR 3 se limita a reproducir exactamente BR 2. Las posibles discrepancias son, por lo tanto, entre BR I y BR 2/3. Se trata, por lo general, de variantes bastantes raras y demasiado leves como para lograr condicionar fundamentalmente una labor de traducción o un trabajo de análisis que la tenga por objeto. Sin embargo, las hay y permiten pormenorizar las relaciones existentes entre las tres ediciones y FR I. Efectivamente, el cotejo que pude llevar a cabo indica que Franciosini debió de manejar BR 2. Sirvan como botón de muestra estos tres ejemplos ${ }^{17}$ :

CAP. XXIII. Fol. 108v.

MADR 2 / BR 1 ... acordò de hurtar el asno a Sancho Páça, no curandose de Rozinante... (209).

BR 2 ... a Sancho, no curandose... (206).

FR I ... si risoluette di rubbar l'Asino a Sancio, non si curando... (231).

CAP. XXV. Fol. 122v.

MADR 2 / BR 1 ... porque te hago saber, que no solo me trae por estas partes el desseo de hallar al loco... (235).

BR 2 ... de hablar al loco... (232).

FR I ... perche voglio, che tu sappia, che io non vengo per questi luoghi per il desiderio solo di fauellar col pazzo... (260).

CAP. XXVI. Fol. 134.

MADR 2 ... No ay para que conmigo amenazas, que yo no soy hombre que robo ni mato a nadie...

${ }^{17}$ En los pasajes citados MADR 2 y BR 1 concuendan con el grupo valenciano y MADR 3. Por eso califico las variantes de significativas. El único caso que se aleja de este patrón es el del capítulo XXVI (MADR 2/3 = VAL1/2-BAR 617 diverge de BR 1), pero la diferencia no resulta importante para lo que es nuestro objetivo. 
BR 1 ... amenazas, dixo Sancho Pança, que yo.... (257).

BR 2 ... amenazas, dixo Sancho, que yo.... (253, pero por equivocación: 553).

FR I ... Io non hò paura di minaccie, diße Sancio, che io non sono huomo da ammazzare, Nene d'asassinar nißuno... (284).

Se trata de casos que poseen un buen grado de seguridad (sobre todo el del cap. XXV). En todos ellos BR 3 confirma su esencial concordancia con BR 2. Con todo, seguimos abogando por BR 2 por presentar BR 3 algunas discrepancias reveladoras. En mi labor de examen textual pude detectar una:

CAP. IV. Fol. $12 v$.

BR $1 / 2 \ldots$ setenta y tres reales... $(24 ; 25)$.

BR 3 ... sesenta y tres reales... (25).

FR I ... settantatre giulij... (28) [Franciosini no se da cuenta del error en el cálculo matemático y lo repite, mientras que BR 3 enmienda].

El dato es, pues, innovador: Franciosini no sigue, como afirmaba Rius, BR 1, sino BR 2. Sin embargo, no es el único: lo más innovador es que Franciosini no siempre acepta la lectio de BR 2. El cotejo de las variantes divergentes nos señala, en efecto, un caso curioso: el traductor tuvo que utilizar MADR 2 u otra edición de ella procedente. He aquí algunos ejemplos:

CAP. XXXX. Fol. 161v.

MADR 2 ... Puesto que ni el Cura, ni Cardenio quisieron yr con ellos, porque no se le acordasse a don Quixote la pendencia que con Cardenio auia tenido: y el Cura, porque no era menester por entonces su presencia... (161v).

BR 2 ... Puesto que Cardenio no quiso yr con ellos, porque no se le acordasse a don Quixote la pendencia que con el auia tenido: y el Cura tampoco, porque no era menester... (303).

FR I ... benche il Pieuano, e Cardenio non volsero andar con loro, accio Don Chisciotte non si ricordaße del rumore ch'haueua fatto con Cardenio, $\&$ il Pieuano, perche per all'hora non occorreua trouaruisi presente... (341).

CAP. XI. Fol. 236.

MADR 2 ... Y esto es, porq. no ay entre ellos sino quatro apellidos de linages, que desciendê de la casa Otomana...

BR 2 ... que contienden en nobleza con la casa Otomana... (441).

FR I ... e cid nasce, perche tra loro non ci è senon quattro sopranomi di Casate, che deriuano da Casa Ottomâna... (500). 
No cabe duda de que, por lo general, los casos de divergencia también se podrían explicar como ejemplos de un fenómeno de génesis interior al texto italiano, es decir, como una interpretación y corrección del traductor debido al examen del contexto lógico de la narración, como faltas de lectura o como ocasionales despistes acarreadores de omisiones. Sin embargo, los loci critici que acabo de mencionar excluyen claramente esta posibilidad y comprueban, en cambio, la hipótesis de una procedencia exterior.

Desde esta perspectiva nuestro interés se centra específicamente en el grupo valenciano (VAL 1-VAL 2; MIL 610; BAR 617). Para averiguar la posible relación entre este grupo y el texto italiano, realicé el cotejo directo basándome en el aparato crítico de Cortejón que funcionó de útil pauta para detectar los casos más interesantes y asegurar, de esta manera, los resultados que iba a conseguir una visión de conjunto. Como prueba de la dependencia de Franciosini de los testimonios de origen valenciano, consegur reunir unos cincuenta-sesenta casos. El criterio de selección adoptado tiende a considerar exclusivamente los casos de divengencia sustancial que aislan de manera inequívoca el grupo valenciano de los demás testimonios [VAL $112=$ BAR $617=$ MIL 610 resultan distintos de MADR 2 = MADR $3=$ BR 1/2/3]. De este grupo, en los ejemplos que voy a citar tan sólo me limito a mencionar las variantes del ejemplar milanés y de los demás, la lectio de MADR 2. Se pueden detectar dos clases de variantes:

a) Casos de adición. Se trata del fenómeno más frecuente. Nunca desemboca en casos de relieve macroscópico. Actúa, más bien, a nivel microscópico, pero con una difusión muy extendida y evidente. El primer ejemplo pone en evidencia exactamente lo microscópico del fenómeno, mientras que los demás (2.a/b) destacan por la alta concentración de adiciones en la zona textual considerada:

\section{CAP. XLIII. Fol. 264}

MADR 2 ... que por amor de mi uiene a pie, y con tanto trabajo, muerome de pesadumbre...

MIL 610 ... que por amor de mi viene a pie, y con tâto trabajo, para el nunca vsado, muerome de pesadumbre... (601).

FR I ... che per amor mio và a piedi, e con tanta fatica, ch'egli non è auuezzo a fare, mi muoio di disgusto... (558).

2.(a) CAP. XIIV. Fol. 273v.

MADR 2 ... corre Sancho hijo, y saca aqui el yelmo q. este buen hobbre dize ser bazia... 
MIL 610 ... corre Sancho hijo mio, y saca aqui con presteza delante de todos estos caualleros el yelmo que este buen hombre dize ser bazia... (623).

FR I ... corri Sancio figliuol mio, e metti quà fuora prestamente innanzi, a tutti questi Signori Caualieri l'elmo che questo buon uomo dice, che è vn bacino... (579).

2.(b) CAP. XLIV. Fol. 273v.

MADR 2 ... Sâcho fue a do estaua la bazia, y la truxo, y assi como dó Quixote la vio la tomo en las manos, y dixo: Miren vuestras mercedes con que cara... y no el yelmo q. yo he dicho: y juro por la orden de caualleria que yo professo .... ni quitado cosa alguna....

MIL 610 ... Sancho fue a do estaua la bazia, o yelmo de Mambrino, como su amo dezia, y la truxo, y asi como don Quixote la vio la tomo en las manos y dixo: Miren las vuestras mercedes señores mios, cô que cara... y no el yelmo de Manbrino que yo he dicho: y juro por la orden de caualleria andante que professo...ni quitado de ninguna suerte cosa alguna... (624).

FR I ... Sancio ando dov'era il bacino, o l'elmo di Mambrino, come il suo padron diceua, e glielo porto, e quando Don Chisciotte lo uedde, lo pigliò in mano, e disse. Guardino di grazia le Signorie vostre Signori miei con che faccia...e non l'elmo di Mabrino, che ho detto... e giuro per l'ordine di Caualleria errante, che professo... ne leuato da nissuna banda, cosa veruna... (579).

b) Casos de lectio distinta. Un ejemplo:

CAP. XXXIX. Fol 230v.

MADR 2 ... siguiesse vno de los caminos que le dire...

ML 610 ... siguiesse vno de los caminos que le diere... (527).

FR I ... seguitasse una delle tre strade, ch'io gli dard... (489).

El cotejo prueba, pues, que directa o indirectamente Franciosini tuvo que echar un vistazo a una de las ediciones valencianas. No faltan, por supuesto, casos en que el traductor italiano se aleja claramente de la lectio de este grupo, pero la divergencia, en ese caso, se debe a la precedencia que Franciosini da a la voz de la edición de Bruselas. Tampoco resulta ilegítima, de considerar los casos de por si mismos, la sospecha de la libre intervención del traductor, pero la existencia de variantes que comprueban la presencia en el texto italiano por lo menos de MADR 2 y, sobre todo, el conjunto de adiciones presentes en la traducción no pueden sino comprobar nuestra afirmación.

El paso sucesivo de nuestra investigación consistiría en averiguar cuál edición del grupo valenciano hubo de seguir en concreto 
Franciosini. Cabe, por lo tanto, profundizar las relaciones genéticas entre los testimonios del grupo. Las dos ediciones valencianas siempre han despertado muchas polémicas en los filólogos cervantinos, que acabaron dividiéndose en los partidarios de la existencia de una sola edición (Rius, Fitzmaurice-Kelly...) y en los que, en cambio, reivindicaban la existencia de unas variantes lo suficientemente fehacientes como para demostrar que se trataba de dos ediciones. Cortejón tomó parte en esta polémica y, partiendo de las cinco discrepancias detectadas por Salvá que Rius copia y califica de leves (Rius: I, 10), realizó por primera vez el cotejo completo de ambas ediciones. Consiguio, de esta manera, detectar 135 discrepancias ${ }^{18}$. (Cortejón: I, lxxii-bxxxii) y, a raíz de ello, concluyó que se trataba de dos ediciones distintas que no correspondían a una misma tirada y que, además, la mayor corrección y pureza estaba a favor de VAL 2.

De las 135 variantes detectadas por Cortejón me detuve tan sólo en las «variantes notorias», es decir, en las que por sus implicaciones semánticas pueden indicar el testimonio del grupo valenciano que Franciosini tuvo que utilizar. Los ejemplares utilizados fueron los de procedencia barcelonesa, que ya hemos mencionado, los cuales difieren ligeramente de los resultados que consiguió Cortejón. El cotejo entre los loci critici considerados y los casos correspondientes que se pueden encontrar en la edición de Barcelona de 1617 y en la edición milanesa de 1610 dio los siguientes resultados. BAR 617 resulta seguir en la mayoría de los casos la lectio de VAL 1. Las pocas veces en que se aleja de ella sigue VAL 2 o presenta una variante independiente. La edición milanesa sigue, en cambio, VAL 2 excepto un solo caso.

Ahora bien, resulta que Franciosini tuvo que recurrir a un ejemplar del gnupo valenciano que siguiese VAL 2. Lo indica, aunque no de una forma intrínsecamente fehaciente, los casos detectados al cotejar los versos intercalados en la narración, los cuales, por venir directamente en el texto italiano en lengua española, desempeñan un formidable papel estratégico ${ }^{19}$. Entre las dos posibles opciones (VAL 2 o MIL 610) nuestra elección va a favor de la edición milanesa. Aboguemos por ella por razones históricas y geográficas, es decir, porque MIL 610 era muy probablemente una de las

18 A decir verdad, son 136. GrvaNel, quien es de igual opinión que Cortejón, descubrí una discrepancia más en el prólogo: respecto en VAL 1 por respeto en VAL 2. A este prop6sito, véase su prologo a la Iconografía de las ediciones del Ouijote de M. Henrich. Barcelona, 1905, p. xii [la noticia y la referencia bibliografica están sacadas del catálogo de Serís, p. 49].

19 Un ejemplo. CAP. XIV: VAL 1/BAR 617....ya que es mas libre...; VAL 2/MIL 610 ...y que es mas libre...; FR I... Y que es mas libre...(116). 
ediciones más asequibles para Franciosini ${ }^{20}$, como por razones exclusivamente filologicas. He aquí, en efecto, algunos casos, significativos justamente porque la lectio de MII 610 diverge de la de todos los demás testimonios y constituye la opción de traducción adoptada por Franciosini:

CAP. XXXX. Fol. 235.

MADR 2 ... y no sé si vino en libertad...

MIL $610 \ldots$ y no se uino en libertad... (537).

FR I ... e non uêne in libertà... (498).

CAP. XLIII. Fol $261 v$.

MADR 2 Donde se cuenta la agradable historia del moço de mulas, con otros estranos acaecimientos en la uenta sucedidos.

MIL 610 Donde se cuenta la agradable historia del moço de mulas, con otros estranos acaecimientos en la uentana sucedidos. [Sin embargo, en la Tabla está escrito aen la uenta sucedidos»] (597).

FR 1. Nel quale si racconta la piaceuole historia del vetturino, con altri strauaganti casi, occorsi alla finestra. (554) [La concordancia con MIL 610 se repite en la Tauola de Capitoli].

A la luz de lo que hasta aquí hemos venido comprobando no nos parece arriesgado disentir de Rius, quien mantiene la procedencia de la traducción italiana de la edición de Bruselas de 1607. La equivocación no radica sólo en que FR I se basa en la segunda edición de Bruselas, sino, especialmente, en que Rius mantiene la tesis de una única procedencia del texto de Franciosini. El cotejo cruzado que hemos llevado a cabo descarta la hipótesis de la existencia de una edición que mezclaría las variantes del grupo de Bruselas con las del grupo valenciano y avala, en cambio, la de una intersección de los dos ejes genéticos de manos del mismo traductor. Franciosini, según esta hipótesis, trabajaría contemporáneamente con los dos testimonios de las dos líneas genealógicas, utilizando uno de éstos como texto básico y el otro como texto de apoyo, o bien pudo recurrir al segundo testimonio sólo en una fase sucesiva.

A este propósito, sería interesante estudiar la concentración de las variantes de BR 2 y MIL 610 aceptadas por Franciosini, es decir, ver de qué manera y con cuáles proporciones se distribuyen a

20 Un dato biográfico significativo al respecto: en la edición romana de 1638 los Dílogos apazibles se publican con una nueva dedicatoria en español dirigida *Al Sefhor don Cosme Marques de Quiani y Maese de Campo en le Estado de Milan por su Magestad Catolica.» 
lo largo del texto italiano, para averiguar cuando el traductor empieza a utilizar una u otra edición (en el supuesto caso de una sucesión cronológica) y/o (en el supuesto de un trabajo que utilice contemporáneamente dos textos) para descubrir cuál es la posición jerárquica y el papel que las dos ediciones desempeñan. Sin entrar en cuestiones demasiado específicas, me limitaré a fijar algunas líneas de tendencia según se puede deducir del examen filológico que realicé directamente siguiendo la pauta del aparato crítico de la edición de Cortejón. Por lo general se puede observar que BR 2 funciona de substrato textual grosso modo hasta los capítulos XXVIII-XXX y llega a su cumbre en correspondencia con el episodio del robo del asno; a partir de dichos capítulos BR 2 cesa de funcionar como hipotexto referencial y se nota la progresiva prevalencia de la edición milanesa que alcanza su apogeo en los capítulos XL-XLV. Aun teniendo en debida cuenta la posibilidad de la intervención autónoma del traductor, se observan fenómenos de transgresión, así que se puede tropezar con casos en que Franciosini sigue la lectio de MIL 610 antes de los mencionados capítulos y viceversa. Esto parecería excluir la hipótesis de una fractura cronológica en la labor de traducción, aunque, claro está, no se puede afirmarlo a ciencia cierta (posibilidad de controles posteriores). La línea de división no es, por lo tanto, rígida, pero consigue igualmente reflejar una diferencia y una tendencia reales.

En cuanto a la posición jerárquica ocupada por las dos ediciones, resulta difícil descubrir cuál de los dos testimonios puede considerarse como texto básico o, incluso, si una de las dos ediciones logra reunir semejantes requisitos. De todas formas, lo cierto es que la modalidad de distribución de las variantes examinadas rebaja la importancia que se atribuía al grupo de Bruselas porque consigue romper un régimen de monopolio genético, $y$, al mismo tiempo, impide calificar la edición milanesa de simple texto de apoyo.

El dato es interesante, y por lo menos por dos razones. En primer lugar, el descubrimiento vale mucho más que una simple cita para comprobar la discreta difusión de la edición milanesa en Italia, por lo menos en la primera mitad del siglo xvn. En este perído la presencia cultural española fue, en efecto, bastante sólida: Italia, si se excluyen los Países Bajos, era el solo país fuera de la Península Ibérica en que se editaban obras en castellano. Milán era un centro editorial de primera magnitud para la difusión de las obras de Cervantes. En las primeras décadas del siglo se publicaron en lengua original una edición del Quijote (1610), las Novelas Ejemplares (1615) y el Viaje del Pamaso (1624). Estas obras estaban dirigidas a un público aristocrático muy aficionado a la cultura española. Que ésta fuera recibida tan sólo como una moda literaria 
y que, por tanto, no pudiera ejercer una real influencia en la cultura italiana, en este caso concreto, no tiene mucho relieve. Lo importante es notar, más bien, que, de una forma u otra, estos libros se leían y conseguían cierto éxito ${ }^{21}$, lo cual, por otra parte, puede contribuir a explicar el fracaso editorial de la traducción de Franciosini ${ }^{22}$.

En segundo lugar, la convivencia de BR 2 y MIL 610 en el texto de Franciosini echa luz sobre las directrices por las que se realizaba el comercio librero en Europa y, más concretamente, sobre la existencia de privilegiados cauces de comunicación que ponían en directo contacto el Ducado Milanés con los dominios de los Hasburgos en el norte de Europa ${ }^{23}$. El dato presenta cierto interés

${ }^{21}$ La dedicatoria All'Ill.mo Señor Conde Vitaliano Vizconde de la edición milanesa posee, al respecto, un llamativo valor documental y permite definir el tipo de público al que el texto cervantino se dirigia: «Cumple a los Grandes, como lo es V.S. Illustriss. el entender todo genero de lenguas principales con las quales se han de tractar los mayores negocios, q. en discurso de tiempo se les puedan ofrecer. Y hauiendo nosotros sabido, que entre los mas graues estudios, en que V. S. Illustriss. passa su pueril edad, tiene a las vezes gusto de la Lêgua Castellana, agora hecha muy familiar a los Caualleros de esta Ciudad; tan noble, por esta razon nos atreuemos a dedicar a V. S. Illustriss. el libro Español del Ingenioso Hidalgo Don Quixote de la Mancha, que de nueuo hauemos impresso, sin hazerlo traduzir en lengua Toscana, por no le quitar su gracia, que mas se muestra en su natural lenguage, que en qualquiera trasladado.... Por otra parte, varias son las noticias que indican que el Quijote era objeto de lectura. Agostino MAscardi en sus Discorsi morali sulla Tauola di Cebete Tebano (1627), demuestra sin lugar a dudas haber leído la versión original, porque hace alusión a los versos preliminares: «No aparece libro desgraciado y ridículo que no ofrezca por delante una copiosa vanguardia de sonetos, de epigramas, de elogios, con la firma de clarísimos autores en descomunales alabanzas del compositor... por lo que no fué sin sagacidad que el autor de la vida de $D$. Quijote, mirando a la vanidad de tales ingenios que se saturan de aire, después de haberlos exhortado a componer en su alabanza lo que más querían, con atribuir las composiciones al preste Juan de las Indias o al emperador de Trapisonda, una gran bandada de sonetos recoge escritos en su elogio - si la memoria del libro que no tengo me asiste fielmente- de Amadís, de D. Belianís, de Orlando y de semejantes paladines. (E. Mele, «Nuevos datos sobre la fortuna de Cervantes en Italia en el siglo XVI», Revista de Filología Española, VII, 3, 1921, pp. 281-82).

22 El texto de Franciosini cuenta tan sólo con tres ediciones a lo largo del siglo xvn. El cotejo con Francia es aplastante: la traducción de Oudin y Rosset tiene la primacía cronológica (1614/1618), se reimprimió desde 1625 hasta 1700 quince veces e incluso tuvo que competir con la traducción de Filleau de Saint-Martin que a partir de la segunda mitad del siglo (1677) circulaba en Francia. En el mismo año en Italia la traducción de Franciosini salía tan sólo por tercera vez.

${ }^{23}$ La edición romana del texto de Franciosini (1677) estaba adornada con ocho grabados copiados de los de la edición de Bruselas de 1662 (Giv.: I, 132). Por otra parte, La traducción de la Picara Justina que Barezzi realizó en 1624-25 adopta por texto-fuente la edición de Bruselas de 1608 (EliSA ArAgONE, «Barezzo Barezzi, stampatore e ispanista del Seicento». Rivista di Letterature Moderne e Comparate, 
y adquiere especial importancia a la hora de averiguar los casos de interferencia que se pueden producir a lo lango del eje horizontal de nuestro esquema genético.

\section{El *Caso OUdin»}

Me estoy refiriendo, por supuesto, a los posibles fenómenos de mediación por parte de otras traducciones contemporáneas del Quijote, y, más concretamente, al papel que pudo ejercer la traducción que de la obra cervantina hizo el francés César Oudin en 1614. El planteamiento del problema resulta legítimo por razones tanto de carácter general como de carácter más específico. En primer lugar, los casos de traducciones italianas de obras españolas realizadas por medio de la mediación francesa no son en absoluto aislados y comprueban una vez más el provincianismo que caracterizo la recepción de la cultura española por parte de la italiana. Tan sólo me limito a recordar, al respecto, el caso de la traducción de las Novelas Ejemplares (Venecia, Barezzi, 1626), cuyo autor, Guglielmo Alessandro de'Novelieri Clavelli, resulta ser un francés que conocía bien la lengua italiana ${ }^{24}$. En segundo lugar, desde una perspectiva más específica, el problema cobra aún más sentido, porque en la producción de Franciosini las obras de Oudin funcionan como

XIV, 4 diciembre de 1961, p. 300). Completa estos datos, y los explica, un llamativo pasaje de Dorini:

all Seicento rappresenta un periodo di decadenza per l'arte del libro in Italia, dove allora affluiscono in quantita ragguandevole $i$ libri stampati all'estero e specialmente la grande produzione dei Paesi Bassi, i quali tengono in quest'epoca il primato nel campo del commercio librario. Cio fu un naturale effetto del risorgimento politico ed economico di quei popoli e del fiorire degli studi nelle loro Universita, fra le quali primeggiava quella di Leida che era frequentata da stranieri di ogni Paese... La guerra dei trent'anni aveva però segnato un arresto nella produzione e nel commercio librario in Germania. Nel 1635 gli editori tedeschi mandarono alla Fiera di Francoforte e di Lipsia soltanto 286 libri nuovi di fronte ai 1358 esposti nel 1613; e fu questo regresso della libreria tedesca che permise agli Olandesi di aumentare la produzione e di estendere maggiormente il loro commencio.» (UMBERTo DORIN, Breve storia del commercio libnario. Milán, Mondadori, Enciclopedia del Libro, 1938, pp. 83; 87-88).

24 Estudian las relaciones entre la traducción italiana y la francesa realizada por F. de Rosset y D'Audigier: Grorge Huinsworth, aLes Nouvelles Exemplaires de Cervantes en Italiex, Bulletin Hispanique, XXXI, 1929, pp. 143-147; JosePH FucrllA, «An Italian Version of Cervantes' "Gitanilla" (From Plagiarism to Translation)», en J. Fucnu, Superbi Colli. Roma, Carucci, 1963, pp. 193-207. Otras noticias, especialmente sobre la actividad lexicografica de G. A. Noviliers Clavel, da A. Galunu, Contributi alla storia della lessicografia..., pp. 293-302. 
una especie de hipotexto referencial que nuestro autor llega incluso a seguir al pie de la letra en una forma de descarado plagio ${ }^{25}$. Dependencia, por otra parte, avalorada por la afinidad de los papeles que los dos autores desempeñaron, aunque en contextos políticos distintos: Oudin actuando de secretario e intérprete al servicio del rey Enrique IV y Franciosini, de profesor en las Universidades de Pisa o Siena, es decir, dentro del más dividido contexto político italiano ${ }^{26 .}$

${ }^{25}$ A. Gallina considera el Thrésor de deux langues françoise et espagnolle de Oudin (1607) como una de las cuatro fuentes del Vocabolario de Franciosini (A. Gallina, Contributi alla storia alla storia della lessicografia..., p 271). Blanca Perinan fija con exactitud la influencia que ejerció la Grammaire et observations de la langue espagnole (1597) en el planteamiento de la Grammatica y señala un caso de ciego servilismo por parte de Franciosini, quien, al definir el adverbio, se deja engahar por el parecido de la palabra francesa nombre por la que Oudin indicaba los adverbios de número y la traduce varias veces con el término equivocado nome (B. PERIRAN, «La "Grammatica"...», p 228). Finalmente, en lo referente a los Dialogos $M$. de Riquer demuestra que proceden de la reedición de la homónima obra de Oudin de 1622 (M. DE RIQUER, «La obra...», p. 26) y Meregalli señala otro caso de ciega fidelidad (Franco Meregalli, Storia delle relazioni letterarie tra Italia $e$ Spagna. Venecia, Libreria Universitaria, 1967, Parte II, fasc. 3, p. 31).

El parecido entre los dos lexicógrafos ha dado pie para acusaciones de plagio y de provincianismo cultural por parte de la generalidad de los hispanistas italianos (por ejemplo: E. Mele, «Uno spagnolista...», p. 178; «Tra i grammatici...», p. 28; F. Meregalli, Storia delle relazioni..., p. 31). No cabe duda que un punto de verdad en estas afirmaciones lo hay. El descano con el que Franciosini usa las fuentes sin citarlas y el paralelismo entre la mediación cultural francesa y la oscilación, en el ámbito de la política, entre Francia y España típica de todos los estados italianos en el siglo XVI, en cierto modo, vienen confirmándolo.

${ }^{26}$ Doy a continuación una breve bibliografía acerca de este hispanista francés que fue lexicógrafo y gramático a la vez que editor y traductor. Noticias biográficas sobre César Oudin se encuentran en: LouIS MORERI, Le Grand Dictionnaire historique,... Parfs, ed. 1747, t. VI, p. 536; A. JAL, Dictionnaire critique de biographie et d'histoire. Paris, 1867, p. 928; A. Morel Fatio, Ambrosio de Salazar et l'étude de l'Espagnol en France sous Louis XIII. Par's, 1901, cap. II-III (pp. 85-186), en el que se profundiza la polémica entre este gramático español que vivia en Francia y el mismo Oudin. Da puntual noticia del viaje de Oudin a España y Portugal ArTuro FARINeU en Viajes por España y Portugal... Florencia, Reale Accademia d'Italia, 1942, t. 11, pp. 15, 33, 49-50. Estudia un curioso caso de plagio Louis CoOPER, «Girolamo Vittori y César Oudin: un caso de plagio mutuo», Nueva Revista de Filología Hispánica, XIV, 1960, pp. 3-20. Resume las noticias biográficas que tenemos sobre este hispanista francés y se detiene sobre todo en su labor de traductor los ya citados estudios de MAURICE BArdon e Isolina SANChez Rrguerra (ver nota 3), además de las mencionadas contribuciones de GrRman Colon (ver nota 1). La misma SANChez Reguriru analiza la obra del hispanista francés desde el punto de vista de la fonética histórica en «La fonética en la obra y en la época de César Oudin», Verba, VI, 1979, pp. 43-75. Igual perspectiva de análisis adopta Amudo Alonso en De la Pronunciación Medieval..., especialmente t. I, pp. 169-180, 212-15; t. II, pp. 237-38. 
Plantea el problema, impulsado por una legítima sospecha, Meregalli:

«El mismo Quijote fue traducido al italiano y publicado en 1622-23 por el citado profesor de español Lorenzo Franciosini: César Oudin lo habia publicado en francés en 1614, y su traducción se reeditó en 1616, 1618, 1620: cuatro veces, antes que en Italia se decidiesen a publicarlo en edición italiana. Si tenemos en cuenta que el benemérito y mediocre profesor había aprovechado los Diblogos apazibles de César Oudin para su texto bilinguie destinado al estudio del idioma, y lo habra aprovechado de manera tan pasiva que al hablar de Pamplona dice que es abella gente, e non poco affezionata alla nostra nazion francesex, podemos asegurar que la traducción del mismo Quijote se debe al ejemplo francés.» ${ }^{27}$

Aparte algunas imprecisiones cronológicas ${ }^{28}$, creo que la afirmación, por más legítima que pueda parecer por las premisas, tan aplastantes, en que se apoya, no consigue escaparse de cierto frenesí deductivo y acaba convirtiéndose en una generalización realmente no muy clara. ¿En qué consiste, en efecto, la deuda de Franciosini con respecto a la obra de Oudin? La pasividad en que se insiste parecería dar la imagen de una labor harto sosa, de una traducción que, sacrificada en aras del provincianismo cultural, va a remolque del ejemplo francés. La afirmación, por lo menos con referencia al conjunto de la producción de nuestro Franciosini, no deja de ser por lo menos en parte verdadera, pero tiene necesariamente que detallarse, de no querer que se convierta en una peligrosa generalización. Y no sólo porque, por más consonancias que pueda uno encontrar entre una obra y su contexto general, siempre hay que someter el juicio a un control específico de carácter filológico, sino sobre todo porque el mismo contexto histórico-cultural puede sufrir correcciones a la luz de cierta diacronía que dinamiza y matiza las tesis demasiado generalizadoras. Cabe recordar, al respecto, que el relevo entre Francia y España se realizó a partir de la segunda mitad del siglo, en coincidencia con una reacción antibarroca, y que en las primera décadas del siglo XVI la presencia cultural española siguió manteniendo su fuerza y prestigio ${ }^{29}$.

27 F. Meregalu, «La literatura española en Italia en el siglo XVI», Annali della facoltd di Lingue e Letterature Straniere di Ca' Foscari, X, 1-2, 1971, p. 181.

28 La fecha * 1622-23 * es con muchas probabilidades una falta de imprenta en lugar de 1622-25, la de 1618 parece ser, en cambio, un malentendido, porque en aquel ańo se publicó la traducción de la segunda parte del Quijote realizada por Rosset y no salió ninguna edición de la obra de Oudin. En realidad, la traducción de la primera parte del Quijote salió por primera vez en 1614 y se reeditó en 1616, 1620, 1625 (an̂o de la muerte de Oudin) y 1632. A partir de la sucesiva edición (1639), salió unida a la traducción de Rosset (pero ver también GIV.: I, 83).

29 . Durante tutta la prima meta del secolo, un'analisi delle traduzioni ci dice che il romanzo francese fu conosciuto da noi attraverso due soli autori: d'Urfé, 
La sospecha de Meregalli resulta, por lo tanto, lógica, pero no deja de ser peligrosamente mecánica a la hora de examinar casos o textos concretos. La procedencia múltiple del texto de Franciosini, en cuanto testimonio de una recepción directa e, incluso, autóctona de la obra maestra de Cervantes por parte de Italia, echa una luz mucho más positiva sobre la figura y los méritos de nuestro autor y aleja de él toda sospecha de mera pasividad que no sea pasejera.

Ahora bien, lo que me propongo demostrar es justamente la independencia, al menos a nivel filológico, que Franciosini mantiene con respecto a lo que es, sin lugar a dudas, un firme punto de referencia para él. Mi cotejo no es en absoluto agotador. Es más: según el enfoque adoptado el texto de Oudin nunca consigue alcanzar la dignidad de obra autónoma, sino simplemente la de un negativo. Dicho de otra forma: lo que esencialmente se va a demostrar no es lo que es la traducción de Oudin, sino lo que es cierto que no es.

Los testimonios que son objeto de nuestro cotejo filológico son las ediciones de 1614,1616 y 1620, es decir, las que preceden la publicación de la traducción de Franciosini. De ellos manejo los ejemplares custodiados en la Biblioteca de Catalunya que indico respectivamente por OUD 1 (Sign.: Cerv. Vit. I-16), OUD 2 (Cerv. Vit. I-28) y OUD 3 (Cerv. 19-III-11). Se trata de ediciones por lo general casi coincidentes. ${ }^{30}$.

Según Rius (I, 206) y Givanel (I, 29) Oudin utilizó para su traducción un ejemplar de la tercera edición madrileña de 1608 que probablemente adquiriría en el viaje que hizo por España en 1610 del que trata en el prólogo de su edición de La Galatea ${ }^{31}$. Tal pro-

cosi mal rappresentato; e soprattutto Camus, le cui fortune si estesero dal 1627 fino al principio del secolo xvw....Verso il 1647 comincio in Italia l'ondata delle traduzioni di romanzi francesi. Quello stesso anno, per la prima volta una pièce di teatro che era il Cid, veniva tradotta in italiano... (BenedeTta Papasogli, «Il romanzo francese barocco in Italia. Bibliografia en Micromégas, 2-3,1978, pp. $115,118$.

${ }^{30}$ Según Rius (RIUS: I, 205-207), las tres ediciones coinciden, por más que en la presentacion $A v$ lecteur se califique la segunda de «reuué, corrigée et purgée d'vne infinité de fautes qui s'estoient coulees par mesgarde en la premierex. Givanel (Grv.: I, 29-30; 49-50; 74-75) profundiza el cotejo filológico y concluye que los tres testimonios difieren desde un punto de vista tipográfico, que la segunda edición de 1616, si no presenta todas las correcciones que promete en el frontispicio, sí tiene algunas $y$, finalmente, que la de 1620 sigue el texto de la primera excepto alguna que otra palabra.

${ }^{31}$ El viaje a España y el contacto con la obra cervantina no es algo casual, sino buscado por Oudin. El hallazgo de La Galatea, según se desprende del problogo en el que Oudin imagina que Galatea escribe una carta a las damas francesas (1611), parece ser lo más importante, tanto es así que, si no la encuentra en Castilla, con tal de dar con ella, Oudin la busca en Portugal, aunque luego la halle llena 
cedencia, muy importante desde nuestro punto de vista porque remancaría la lejanía del texto de Franciosini del de Oudin, quedará clara tanto indirecta como directamente. En primer lugar, nuestro cotejo apunta a poner de relieve que Oudin no conoció ni utilizó los textos a los que recurrió el traductor italiano, es decir, BR 2 y MIL 610. Hago, por tanto, referencia a los pasajes citados anteriormente ( $\mathrm{y}$ a otros también) y los voy a cotejar con las tres ediciones del texto de Oudin, del que no tengo en cuenta los cambios tipográficos, y la de MADR 3 .

Ya de entrada queda patente y comprobada la distancia de la obra de Oudin de BR 2. Como botón de muestra valgan estos dos ejemplos:

CAP. XXV. fol. 122

MADR 3 ...y de aqui adelante entremetete en espolear a tu asno...(107v)

BR 2... y de aqui adelante entremetete en servir a tu amo...(231)

FR I....\& per l'auuenire attendi a seruire il tuo padrone...(260)

OUD $1 / 2 / 3 \ldots . . . \&$ d'ici en auant mesletoi de piquer tô asne...(294)

CAP. XXXVI. Fol. 214

MADR 3... y a vno dellos le pregunto lo que ya desseaba... (188r)

BR 2... y a vno dellos le pregunto lo que saber desseaba... (400)

FR I....\& a vn di loro dimando quanto egli desideraua sapere...(454)

OUD 1/2/3....\& demanda a l'vn d'eux ce qu'il desiroit...(501)

En estos dos casos MADR 3 coincide con MADR 2, de que siempre he indicado el folio. La pequeña muestra que a continuación sigue evidencia aún más rotundamente la distancia entre las dos traducciones, porque comprueba de manera inequívoca y positiva la procedencia de la traducción francesa de MADR 3 (MADR 2 distinto de MADR 3 y FR I = BR 2). Se trata de loci critici que no se sitúan solamente en la zona estratégica del episodio del robo (cap. XXIII):

CAP. XXIII. Fol. 112.

MADR 2 ... y siguiole Sancho con su acostumbrado jumêto...

de errores. Probablemente la principal razón que Oudin tenía para publicar La Galatea de Cervantes se debería al hecho de que ya tenía la intención de dar a conocer el Quijote en lengua francesa a un público que de la obra maestra cervantina ya conocía los episodios sentimentales. (I. SANCHEz REgUERA, «El hispanista francés Cesar Oudin...», pp. 117-118). 
MADR 3 ... y siguiole Såcho a pie, y cargado, merced a Ginesillo d. Pasamôte...(98v)

BR 2....y siguiole Sancho a pie consolado de la perdida II de su jumento con la esperança de los tres pollinos... (213-14).

FR I ... e Sancho lo seguito a piedi; consolato della perdita del suo giumento per la speranza de tre polledri... (238).

OUD 1/2/3. \& Såcho le suiuit a pied \& tout chargé qu'il estoit, Dieu merci \& Ginesillo de Passamont... (270).

Sino también en otras zonas textuales igualmente fehacientes:

CAP. XIII. Fol 260v.

MADR 2 ... y el le puso anchas manos en los pechos...

MADR 3 ... y el le puso las manos en los pechos... (228r).

BR 2 ... ambas manos.... (485).

FR I ... \& ei gli messe ambe le mani al petto... (551).

OUD $1 / 2 / 3 . .$. qui luy mit les mains contre l'estomach... (608).

Asimismo, queda también excluida toda forma de presencia de la edición milanesa en la traducción de Oudin. Se trata, sobre todo, de casos de adición (a) o de lectio distinta (b) que se pueden encontrar grosso modo a partir de la cuarta parte del Quijote. Me limito a citar tan sólo dos de tantas variantes que a lo largo de mi labor de investigación pude controlar. En ellas la edición madrilena de 1608 no diverge de MADR 2 de que indico el número del folio:

a. CAP. XIIV. Fol. 273v.

MADR 3 ... corre Sancho hijo, y saca aqui el yelmo que este buen hôbre dize ser bazia... (239r).

MIL 610 ... corre Sancho hijo mio, y saca aqui con presteza delante de todos estos caualleros el yelmo que este buen hombre dize ser bazia... (623).

FR I ... corri Sancio figliuol mio, e metti quà fuora prestamente innanzi, a tutti questi Signori Caualieri l'elmo che questo buon uomo dice, che vn bacino... (579).

OUD 1/2/3 ... cours Sancho mon fils, \& apporte icy cest anmet que ce bon homme dir estre vn bassin... (638; falta de paginación en OUD 3: 380).

b. CAP. XXODX. Fol. 235.

MADR 3 ... y no se si vino en libertad... (206r). 
MIL $610 \ldots$ y no se uino en libertad... (537).

FR I ... e non uêne in libertà... (498).

OUD $1 / 2 / 3 \ldots$ \& ne suis pas certain s'il eschappa, biê que i'estime qu'ouy... (548).

Por los datos hasta aquí reunidos queda, por tanto, claro que Oudin no sigue los dos textos-fuentes de que se sirvió Franciosini, sino la tercera edición madrileña ${ }^{32}$. A decir verdad, no deja de haber ejemplos en que él actúa de forma independiente y se aleja de su texto-fuente para enmendarlo. Entre ellos se pueden sí detectar pasajes textuales en que las correcciones coinciden con la lectio de los textos de partida de la traducción italiana ${ }^{33}$, pero también se dan casos en que la corrección no coincide con la lectio de ningún otro testimonio, de forma que la independencia de la traducción francesa del texto italiano queda una vez más comprobada. Considérese este ejemplo en el que la corrección equivale por exactitud semántica a la lectio de $B R 2$, pero en definitiva resulta totalmente personal ${ }^{34}$ :

CAP. XXV. Fol. 121.

MADR 3 ... porque departiera yo con mi jumento lo que me viniera en gana... (106v).

${ }^{32}$ Recuerdo otros dos ejemplos en los que salta a la vista que Franciosini, en cambio, no sigue de ningún modo MADR 3. En ellos cito FR I, MADR 3 y OUD 1/2/3. FR I sigue BR 2 o MIL 610, los cuales coinciden con MADR 2 de que indico el número de folio. Se trata de un caso de omisión: CAP XIV Fol. 57v. FR I....Ella ha mostrato con manifeste, \& sufficienti ragioni...(123); OUD $1 / 2 / 3$....Elle a demonstré auec raisons euidents... (138); MADR 3... con claras razones... (50v). Y de un caso de modificación semántica: CAP. XXV. Fol. 130 FR I....Data nelle viscere di Sierra Morena a ventidua d'Agosto di questo present'anno...(276); OUD 1/2/3....fait és entrailles de la Sierra Morena, le vingt-septiesme Aoust de la presente annee... (312); MADR 3... a veinte, y siete Agosto... (114v).

${ }^{33}$ Véase este ejemplo en que la omisión acerca el texto francés a BR 2. CAP. XXV. Fol. 120v MADR 3... mando a Sancho que le siguiesse, el qual lo hizo con su jumento, de muy mala gana... (106v); BR 2... mando a Sancho que le siguiese, el qual lo hizo de muy mala gana... (232); FR I....disse a Sâcho, che lo seguitasse; il che egli fece molto mal volentieri...(257); OUD 1/2/3....commanda à Sancho qu'il le suiuit, ce qu'il fit de fort mauvaise volonté...(291).

34 Por otra parte, no deja de haber pasajes textuales en que, en cambio, es el propio Franciosini quien manifiesta su independencia. Véase, por ejemplo, la enmienda del epigrafe del cap. XI. El traductor italiano se percata de la falta de pertinencia temática (el episodio de los Yangúeses se relata en el cap. XV) y corrige, aunque, a decir verdad, su labor de corrección se queda a medias, porque la aventura del vizcalno ya se habla acabado. CAP. X. BR 2/MADR 3. de lo que mas le auino a Don Quixote con el Vizcayno: y del peligro en que se vio, con vna caterua de Yangueses. (67/30v); FR I. Che tratta d'altre cose, che D. Chisciotte passo col Viscaino. (77); OUD 1/2/3. De ce qui aduint de plus a Don Quixote auec le Biscayn du peril auquel il se trouua auec vne troupe de Yangois. (82) 
BR 2 ... porque departiera yo con Rozinante (ya que mi corta vêtura no permitio pueda ser con mi jumento) lo que me viniera en gana... (229).

FR I ... che io potrei dire con Ronzinâte, (già che la mia cattiua disgrazia non ha voluto ch'io possa far questo col mio giumento) quello che mi veniße alla fantasia... (257).

OUD $1 / 2 / 3$... car ie pourrois deuiser $\|$ auec mon asne, si ie l'auois, de ce qui me viendroit en la fantasie... (291-92; OUD 2/3: 292).

El cotejo hasta aquí llevado a cabo no puede, por supuesto, excluir que se baraje la hipótesis de la procedencia múltiple de la traducción de Oudin, es decir, que el traductor francés no se sirviera sólo de MADR 3, sino de otros textos. Sin embargo, sí permite afirmar que, si los hay, no se puede tratar justamente de BR 2 y MIL 610. Los diferentes recorridos que los dos traductores siguen quedan, pues, muy claros. Por otra parte, el texto de Oudin presenta unos rasgos que, ya a primera vista, lo distinguen del de nuestro Franciosini. Basta con recordar tan sólo algunos llamativos casos de omisión que no tienen correspondencia en el texto italiano. Al final de la primera parte del Quixote, por ejemplo, Oudin omite los sonetos cervantinos y se limita, en cambio, a traducir solamente los epitafios que están sobre la tumba de Don Quijote y Dulcinea. Asimismo, en el capítulo XXXIII, al relatar la historia del Curioso Impertinente, suprime sin ninguna razón la estancia del poeta Luis Tansillo y la frase que le sigue referente a la prueba del vaso que Cervantes encontró en Ariosto. De una manera análoga, en el mismo episodio, Oudin elimina el pasaje en que Leonela afirma que Lotario posee no sólo las cuatro S.S.S.S., sino más bien un entero alfabeto.

Con todo, si los datos hasta ahora reunidos permiten demostrar lo lejos que está la traducción francesa de la italiana, no consiguen borrar la sospecha de que pudo haber servido de texto de apoyo colateral. Cabe, pues, pormenizar aún más el cotejo. Nuestra atención se centra en los casos en que filológicamente los textos-fuente de los dos traductores convergen y, no obstante, el resultado final de la traducción equivale a variantes distintas. Los que a continuación se reúnen son unos cuantos casos en que muy claramente el traductor francés acierta mientras que Franciosini cae en faltas a veces garrafales. La lista, desde luego, no tiene la pretensión de fijar una jerarquía de valores que permita juzgar las dos traducciones, pero sí la de reunir unos cuantos casos, significativos justamente porque muy probablemente Franciosini no hubiera cometido semejantes faltas de haber hojeado de verdad el texto francés. Como textos-fuentes de la traducción italiana utilizo BR 2 hasta el capitulo XXX y para los capítulos siguientes MIL 610, a menos que haya 
casos divergentes. Se trata de pasajes que presentan una lectio semánticamente distinta como consecuencia de un malentendido por parte del traductor italiano:

CAP. II.

BR $2 /$ MADR 3 ... Luego bol [via] diziendo... (10; 4v).

FR I ... Poi... si volto dicendo... (10).

OUD $1 / 2 / 3 \ldots$ Incontinêt il se remettoit a dire... (11).

CAP. XXVIII.

BR 2/MADR 3 ... Que Dorotea es tu nombre, Señora? Otra he oydo yo dezir del mesmo nombre... (289; 135v).

FR I ... Io ho udito a lui steßo nominarne vn'altra... (325).

OUD $1 / 2 / 3$... Quoy, ton nom est Dorothee, Madame? I'ay ouy parder d'vne autre qui a le mesme nom... (367).

En casos concretos la falta semántica se debe a un trueque referencial que afecta a los mismos personajes (muchacho-Don Quijote; Cardenio-Fernando):

CAP. III.

BR 2/MADR 3 ... y cô vn cabo de vela que le traia vn muchacho...se vino adonde Don Quixote estaua, al qual mando hincar de rodillas... (22; 10r).

FR I ... \& con vn pezzo di candela che gli portaua vn ragezzo...ando alla stanza di Don Chisciotte, \& arriuati, l'hoste fece inginocchiare il ragazzo... (24).

OUD 1/2/3... \& auec vn bout de chandelle que luy apportait vn petit garçon...il s'en vint la ou Don Quixote estoit, auquel il commanda de se mettre à genoux... (26).

CAP. XXIV.

BR 2/MADR 3 ... quiso la fortuna que [Femando] hallasse $\mathrm{vn}$ dia $\mathrm{mn}$ villete suyo... que... en leyendolo me dixo... (225; 104v).

FR I ... volse la sorte che io trouassi un giomo un suo viglietto.... che subito ch'io lo lessi, mi diße... (252).

OUD $1 / 2 / 3$... 1a fortune voulut, qu'il trouuast vn iour $\mathrm{vn}$ sien billet...qu'en le lisant il me dit... (286).

No faltan, por otra parte, casos de divergencia ocasional. En el capítulo XXVIII, por ejemplo, Franciosini y Oudin concuerdan en la traducción del juego verbal basado en el término cuento: 


\section{CAP. XXVIII}

BR 2/MADR 3 ... Mas por acabar presto con el cuento (que no le tiene) de mis desdichas... (288; 133v).

FR I ... Ma per finir presto questa historia delle mie disgrazie, che non ha fine... (321). La nota al margen reza: «Quí nô si puó alludere col vocabolo spagnolo).

OUD $1 / 2 / 3$... Mais pour bien tost mettre fin au conte de mes malheurs, qui sont sans fin... (363).

Sin embargo, en el capítulo anterior, Franciosini opta por una traducción divergente que nos parece incluso original ${ }^{35}$ :

\section{CAP. XXVII}

BR 2/MADR 3 ... os ruego que escucheys el cuento, que no le tiene de mis desuêturas ... $(265 ; 123 v)$.

FR I ... vi voglio pregare ad ascoltare l'inenarrabil'historia delle mie tribulazioni... (298).

OUD $1 / 2 / 3$... ie vous prie que vous escoutiez le conte qui est infiny de mes disgraces...(336).

Desde luego, éstos no son más que unos pocos ejemplos y el cotejo tendría que extenderse para adquirir mayor dignidad. Sin embargo, creo que ya estos pocos casos son suficientes para alejar de la figura de Franciosini el espectro de la dependencia aniquiladora de su alter ego francés y, por consiguiente, toda acusación de pasivo servilismo.

La independencia, de todas formas, no supone la ignorancia. Oudin funciona de continuo aliciente para Franciosini y, con toda probabilidad, sería el éxito que estaba consiguiendo por aquel entonces la traducción francesa lo que indujo al traductor italiano a emprender la hazaña de enfrentarse con la obra maestra cervantina y a conservar, de esta manera, el paralelismo que caracterizaba su producción con respecto a la de su especimen francés. En cierto modo, tampoco se puede excluir a ciencia cierta que Franciosini conociera la traducción de Oudin y la hubiese podido hojear, aunque, luego, eso sí, acabó siguiendo rumbos distintos.

La imagen de Franciosini se nos vuelve, pues, mucho más positiva y original, porque él se muestra también capaz de actuar dentro del contexto hispano-italiano de una forma directa y personal. Bien mirado, esta independencia no se apoya exclusivamente en los datos filológicos, de por sí muy fehacientes. Cabría preguntarse por qué las deducciones generalizadoras han fallado. En parte se debe

${ }^{35}$ Véase también este ejemplo, en que la divergencia semántica desemboca en un curiosa traducción rica en personal humorismo. CAP. XXXI. MIL 610/MADR 3... es que senti vn olorzillo algo hombruno... (397; 152r); FR I... che io senti vn ododrino spallatico... (367); OUD 1/2/3....c'est que ie sentis vne petit odeur, quasi comme celle d'vn honme... (412). 
a cierto apresurado automatismo que no ha sido capaz de tener en debida cuenta la intrínseca autonomía que el nuevo contexto lingüístico, el italiano, ya a priori aseguraba a la actividad de nuestro traductor. En parte -y esto vale sobre todo por lo que a la producción lexicográfica de Franciosini se refiere - el fallo es debido a la escasa atención que se ha puesto en la interpretación del «robo» como una forma concreta de intercambio cultural. No queremos necesariamente, aunque no sea innecesario, referirnos a las «teorías» de la época y proporcionar la imagen de un Franciosini que juega con il rampino. Tan sólo es suficiente recordar que en el ámbito lexicográfico la transposición de clasificaciones completas y de ejemplos era práctica muy corriente por aquel entonces y que el propio Oudin copiaba y, para remate, con no menor desparpajo que Franciosini ${ }^{36}$. El juicio sobre la originalidad tiene, por tanto, que radicar en el más sutil juego de las afinidades y divergencias que alimenta la posibilidad de la independencia y evoluciones; y esto sobre todo en el caso concreto de nuestro autor, quien, tal vez por alguna que otra deformación profesional, tiende a seguir muy de cerca a sus especímenes anteponiendo una silenciosa y atenta documentación a la manipulación revolucionaria. En cierto modo, Franciosini crece con sus modelos. Oudin no es, por tanto, un simple patrón al que referirse y a veces citar demasiado fielmente, sino también un aliciente para una actividad esencialmente independiente.

Desde este punto de vista, sería realmente provechoso analizar las posibles semejanzas y diferencias metodológicas entre la traducción francesa y la italiana. Los estudios de Bardon e I. Sánchez Regueira concuerdan al juzgar la labor de Oudin. Se trata de una traducción ad litteram, en la que la adherencia textual, a veces ex-

${ }^{36}$ Que este género de despojos fuera bastante frecuente en aquella época lo demuestra el caso de mutuo plagio entre Oudin y Girolamo Vittori, autor de un Tesoro de las tres lenguas española, francesa y italiana (Ginebra, 1609), en el que se detiene LuIs COOPRR [^Girolamo Vittori...», pp. 3-20]. Cooper consigue demostrar que la acusación de plagiario que Oudin dirigió a Vittori en la segunda edición de su Thrésor corresponde sólo parcialmente a la verdad, porque, si bien Vittori copia casi toda la obra de Oudin (1606), añade, sin embargo unos 3.000 artículos nuevos y lo extraño es que casi las tres cuartas partes de estas adiciones se encuentran, a veces modificadas, en la edición de la obra del autor francés de 1616. Es decir: «Oudin plagió a Vittori tan decididamente como éste le había plagiado antes a é, y sin remordimientos algunos, justificando su acción como natural represalia.» (p. 10) Además, ambos ocultan las mutuas deudas: Oudin jamás las reconoce y se limita a acusar públicamente a Vittori y éste, en vez de enorgullecerse de ser el autor indudable de una parte de la obra, prefiere dar vagamente a entender que toda ella le pertenece (p. 7). Por otra parte, el propio Menegalli al mismo tiempo que asegura que kla traducción [italiana] del mismo Quijote se debe al ejemplo francés» anade entre paréntesis: «Pero resulta que César Oudin, por su parte, casi copió sus Dialogos apazibles del ingles Minshev» (F. Mrregnul, «La literatura española en Italia...», p. 181) . 
cesiva hasta el punto de llegar a ser oscura, corre parejas con el minucioso esmero y el escrúpulo exegético, evidente sobre todo a la hora de traducir los giros del idioma de partida. El límite lo subraya Bardon: «à force de vouloir ajuster strictement sa prose à la prose castillane, il ne nous fournit par encroits qu'une sorte de décalque, d'une litteralité fatigante» ${ }^{37}$. Ahora bien, dado que esta fidelidad parece ser un rasgo distintivo también de la traducción de nuestro Franciosini y, además, conformarse con las tendencias y el gusto típico de la época ${ }^{38}$, lo que resultaría de veras muy interesante sería profundizar el cotejo entre las dos traducciones, detectar los códigos de control que gobiernan esta fidelidad y averiguar dónde y cuándo se manifiestan las divergencias. Sin embargo, éste sería argumento para otro artículo que comprobase, a nivel de estrategias traductoras, las diferencias entre el texto italiano y el francés que hemos venido poniendo de relieve según un enfoque filológico. El tema queda, por tanto, abierto y proporciona nuevas posibilidades de investigaciones ${ }^{39}$.

\section{DANTE BERNARDI}

37 M. BARdon, «César Oudin, traducteur...», p. 28.

38 «a traducción inglesa de Shelton -1612-, la traducción francesa de Oudin -1614-, y la italiana de Franciosini - 1622- tienen, con relación a sus lectores, más parecido del que pudiera creerse. Todas tienden a la exactitud literal.» (FranCISCo A. DE ICAZA, El Quijote durante tres siglos. Madrid, Fortanet, 1918, p. 85).

${ }^{39}$ Germán Colón acaba un artículo dedicado a las dos traducciones con una pregunta que nos parece muy interesante al respecto: «Sorprende en nuestras dos nóminas la escasez del binomio hispano-italiano frente a la abundancia del hispano-francés. ¿Apuntará así a un rasgo del estilo más individualista del traductor Franciosini?» (Véase: G. ColóN, «Materiales para el estudio léxico...», p. 93]. Contribuye a dar más crédito a esta hipótesis la actitud metodológica que Oudin mantiene a la hora de poner notas al margen de su traducción. Destacan, sobre todo, su preocupación por citar el exacto contenido del texto cervantino toda vez que su traducción, de una forma u otra, acaba alejándose del texto de partida, y la puntualidad con la que se manifiesta esta preocupación, es claro indicio de un decidido apego a la letra. Un ejemplo sacado del citado artículo de I. SANCHEz REgUeIRA (p. 15): «en el f. 388 dice el texto cervantino: «Y quien soys vos, hermano? que traduce Oudin (p. 378): "et qui estis vos mon amy". Aclara en la nota: "L'Espagnol dit, freure au lieu que nous disons amy ou comperex." Semejantes aclaraciones se encuentran muchas veces en la traducción francesa y el mismo Bardon no puede menos que observarlo y hablar, a proposito de Oudin, no sólo de «diligence minutieuse», sino incluso de «timidité (p. 30). En el texto de Franciosini, en cambio, la nota funciona de recurso metatextual para aclarar pasajes difíciles o declaración de rendimiento ante la imposibilidad de traducción (especialmente en el caso de los juegos de palabras), pero muy pocas veces declara el alejamiento del texto-fuente y nunca cita el correspondiente español. Indicio, este último, de una estrategia de traducción de tipo parafrástico para la que remito a mi tesis inédita citada anteriormente. 